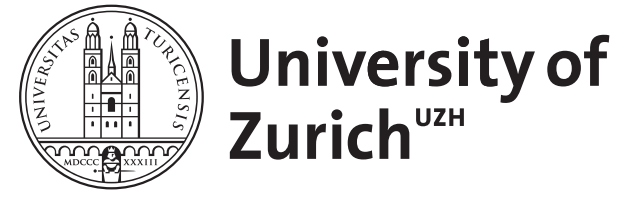
Archive

University of Zurich

University Library

Strickhofstrasse 39

CH-8057 Zurich

www.zora.uzh.ch

Year: 2018

\title{
Chronische Erkrankungen und Spiritual (Self-)Care
}

Peng-Keller, Simon ; Bischoff, A

DOI: https://doi.org/10.1515/spircare-2018-0032

Posted at the Zurich Open Repository and Archive, University of Zurich

ZORA URL: https://doi.org/10.5167/uzh-160827

Journal Article

Published Version

Originally published at:

Peng-Keller, Simon; Bischoff, A (2018). Chronische Erkrankungen und Spiritual (Self-)Care. Spiritual Care, 7(3):241.

DOI: https://doi.org/10.1515/spircare-2018-0032 


\section{Editorial}

\section{Simon Peng-Keller* und Alexander Bischoff \\ Chronische Erkrankungen und Spiritual (Self-)Care}

https://doi.org/10.1515/spircare-2018-0032

Seelsorgliche und gesundheitsberufliche Spiritual Care ist in allen Bereichen der Gesundheitsversorgung gefragt. Das vorliegende Heft widmet sich dem weiten Gebiet der chronischen Erkrankungen. Es gehört nicht zu jenen Gebieten, in dem die Medizin regelmäßig große Erfolge feiert. In den letzten Jahrzehnten wurden viele akute Krankheiten immer besser therapierbar, während die chronischen Gesundheitsprobleme zunehmen. Sie sind heute die größte Herausforderung für ein Gesundheitswesen. Das Gebiet der chronischen Krankheiten ist nicht nur weit und wachsend, sondern auch sehr heterogen. Es umfasst nicht übertragbare Krankheiten (Herzkreislauferkrankungen, Diabetes, Krebs etc.), übertragbare Krankheiten (wie HIV/AIDS oder Tuberkulose), Behinderungen, sowie psychische Störungen (Depression u. a.m.).

Die Bedeutung der spirituellen Dimension mag im $\mathrm{Zu}$ sammenhang mit chronischen Krankheiten weniger deutlich hervortreten am Lebensende. Umso wichtiger ist es, sie in ihrer Vielfalt zu erschließen: als Ressource, als Sehnsucht und mitunter auch als Belastung. Die Einsicht, dass es nicht erst am Lebensende, sondern auch bei chronischen Erkrankungen sinnvoll ist, Spiritual Care in interprofessioneller Zusammenarbeit wahrzunehmen, ruft nach neuen Praxismodellen oder zumindest nach einer Erweiterung bestehender interprofessionelle Ansätze. Müsste beispielsweise die spirituelle Dimension nicht auch in einer multimodalen Schmerztherapie stärker berücksichtigt werden? Ist sie in onkologischen Therapien nicht selbst dann bedeutsam, wenn die Krebserkrankung heilbar sein sollte?
Wir fragen zudem: Ist Spiritual Care wirklich nur auf den Umgang mit der Krankheit beschränkt? Ist sie nicht auch Teil der Behandlung? Hat Spiritual Care auch eine kurativ-therapeutische Seite? Handelt es beispielsweise beim folgenden Fallbericht um eine Form von Spiritual Care?

Eine Ordensfrau, die aufgrund traumatischer Erfahrungen verstummt war, fand im Rahmen einer Psychotherapie wieder zurück zur Sprache. Dabei spielte das Rezitieren von Psalmen eine Schlüsselrolle: „Die einzige Möglichkeit, Worte zu sagen, war das Rezitieren von Psalmen in einer Kommunität. In diesen Psalmen konnte ich Dinge sagen, die ich unter anderen Umständen nicht hätte sagen können (...). Das Psalmenrezitieren hat mir wirklich das Leben gerettet, in dem Sinn, dass ich habe Dinge sagen können, die in mir vor Gott waren, mit Worten, die mir von Gott gegeben wurden und für mich entscheidende Wichtigkeit hatten“ (Fournier 2013: 205).

Ein Beispiel für Spiritual (Self-)Care? Das vorliegende Heft regt mit Blick auf chronische Erkrankungen an, über die Möglichkeiten und Grenzen dessen nachzudenken, was dieser Zeitschrift den Namen gibt!

Simon Peng-Keller \& Alexander Bischoff

\section{Literatur}

Fournier CA (2013) Crise, construction identitaire et vocation. Emprise maternelle. Quand Dieu vient au secours d'une narrativité confisquée. Revue d’éthique et de théologie morale, 276: 183-209. 